\title{
Low Frequency of Leisure-Time Activities Correlates with Cognitive Decline and Apathy in Patients with Parkinson's Disease
}

\author{
Kayoko Miura',2, Shutaro Takashima ${ }^{3 *}$, Mie Matsui ${ }^{1}$, Kortaro Tanaka ${ }^{3}$ \\ ${ }^{1}$ Department of Psychology, Graduate School of Medicine and Pharmaceutical Sciences, University of Toyama, \\ Toyama, Japan \\ ${ }^{2}$ Japan Society for the Promotion of Science, Tokyo, Japan \\ ${ }^{3}$ Department of Neurology, Toyama University Hospital, Toyama, Japan \\ Email: shutaro@med.u-toyama.ac.jp
}

Received 15 June 2014; revised 17 July 2014; accepted 5 August 2014

Copyright @ 2014 by authors and Scientific Research Publishing Inc.

This work is licensed under the Creative Commons Attribution International License (CC BY).

http://creativecommons.org/licenses/by/4.0/

(c) (i) Open Access

\section{Abstract}

Objective: The purpose of this study is to investigate habitual leisure-time activities (physical and non-physical leisure activities) in patients with Parkinson's disease (PD), and to determine any potential benefits of these activities on cognitive functions and emotional symptoms. Methods: Thirty-two patients with PD and 25 demographically-matched healthy controls participated in the present study. Neuropsychological tests (Wisconsin Card Sorting Test, Trail Making Test, Digit Span Test, Verbal Fluency Test, and Japanese Verbal Learning Test), assessment of emotional symptoms, and interviews for confirmation of habitual leisure-time activities were conducted for all participants. Results: Patients with PD significantly showed the lower frequency of both physical and non-physical leisure activities than healthy controls. Compared to patients who engaged in physical leisure activities, patients who did not engage in such activities performed worse on the Trail Making Test (TMT-B, and TMT-B minus A). Moreover, patients who engaged in non-physical leisure activities were less apathetic than patients who did not engage in such activities. Conclusions: Our preliminary study shows that habitual leisure-time activities correlate with cognitive function and emotions in patients with PD. Reducing sedentary lifestyles and promoting habitual leisure-time activities may be helpful for preventing cognitive decline and apathy.

\section{Keywords}

Parkinson's Disease, Physical Leisure Activity, Non-Physical Leisure Activity, Cognitive Function, Apathy

\footnotetext{
${ }^{*}$ Corresponding author.
} 


\section{Introduction}

Parkinson's disease (PD) is a chronic and progressive neurodegenerative disorder that is mainly caused by dopaminergic neuron loss in the substantianigra. The symptoms of PD include tremor, rigidity, bradykinesia, postural instability and non-motor symptoms, such as cognitive impairment, emotional problems, and sleep problems. Patients with PD tend to lead sedentary lifestyles due to a combination of motor and non-motor symptoms. Moreover, in these patients, a vicious cycle of sedentary lifestyle and physical inactivity is unfortunately common. Both a sedentary lifestyle and physical inactivity lead to specific symptoms of PD, for example insomnia, depression, apathy, and cognitive decline [1].

In a study on elderly individuals, cognitive impairment and development of dementia were associated with lifestyles as well as genetic and biological factors [2]. However, we have little evidence linking lifestyles and non-motor symptoms such as cognitive decline and emotional problems in patients with PD. Retired individuals tend to engage in physical leisure activity [3], and participating in leisure-time activities may improve the quality of life for these individuals. In addition, non-physical leisure activity has been shown to have a beneficial impact on cognitive function in the elderly [4]. Thus, elderly individuals should participate daily in both physical and non-physical leisure activities.

In patients with PD, a lack of habitual leisure-time activities may negatively affect cognitive function and emotion, but clear evidence for this is missing. Accordingly, additional studies are needed to clarify whether habitual leisure-time activities influence cognitive function and emotions in patients with PD. The purpose of this study is to investigate habitual leisure-time activities (both physical and non-physical leisure activities) in patients with PD, and to clarify any relationship of these activities with cognitive functions and emotional symptoms.

\section{Methods}

\subsection{Participants}

Thirty-two patients with PD (PD group: 10 men, 22 women) and 25 demographically matched healthy controls (HC group: 9 men, 16 women) participated in the present study. The patients were recruited from outpatient departments at the Toyama University Hospital. The HC participants were recruited from local residents (i.e., members of local community centers). Participants were excluded from the study if they had: 1) a Mini Mental State Examination Japanese (MMSE-J) total score below 25 and 2) a history of any other neurologic disorder and/or acquired brain injury. The two groups did not differ in age (PD: $67.8 \pm 7.4$ years, HC: $66.9 \pm 7.2$ years; $t(55)=-0.49, p=0.624)$, total period of education (PD: $12.0 \pm 1.6$ years, HC: $13.2 \pm 2.6$ years; $t(55)=1.91, p=$ $0.063)$, and gender $\left(\chi^{2}(1, N=57)=0.143, p=0.706\right)$. PD diagnosis and disease severity (Hoehn and Yahr stages: HY) were verified by at least one neurologist. All patients with PD were taking anti-parkinsonian drugs at the time of neuropsychological testing. Doses of dopaminergic medication were converted to L-dopa equivalent daily dose (LEDD). The present study was approved by the Ethics Committee of the University of Toyama, and all participants signed a written consent form before undergoing the assessment.

\subsection{Assessments}

\subsubsection{Habitual Leisure-Time Activity}

All participants were interviewed to determine the presence or absence of habitual leisure-time activities. They were classified as the engagement group or non-engagement group for both physical and non-physical leisure activities. Participants were defined as engaging in a physical leisure activity if they voluntarily performed daily exercises, such as walking, cycling, or participating in sports. Participation in a non-physical leisure activity was determined if subjects voluntarily spent their discretionary time in non-compulsory activities, such as social activities, hobbies, and other activities except exercise.

\subsubsection{Cognitive Function}

We used a battery of neuropsychological tests to assess cognitive functions, such as executive function, attention, processing speed, and memory. The following tests were included in the neuropsychological test battery: Wisconsin Card Sorting Test (WCST), Trail Making Test (TMT), Digit Span Test (DST), Verbal Fluency Test (VFT), and Japanese Verbal Learning Test (JVLT). 


\subsubsection{Emotional Symptoms}

The severity of depressive symptoms was rated using the 17-item version of the Hamilton Depression Rating Scale (HAMD-17). The level of apathy was assessed using the Japanese version of the Apathy Scale.

\subsection{Statistical Analysis}

An independent t-test was used for comparing means between the groups. An appropriate non-parametric test such as Mann-Whitney U-test was applied whenever normality assumptions were not satisfied. Between-group comparisons of categorical variables were performed using $\chi^{2}$ tests.

\section{Results}

\subsection{Physical Leisure Activity}

In the HC group, 21 individuals (84\% of the total) were engaging in a physical leisure activity. On the other hand, in the PD group, only 14 patients (44\%) were doing so. The PD group showed a significantly lower frequency of physical leisure activity than the HC group $\left(\chi^{2}(1, N=57)=9.59, p=0.003\right)$. Participants reported the following physical leisure activities: PD: radio gymnastics, walking, park golf, etc.; HC: park golf, fresh tennis, ballroom dancing, walking, etc. In order to examine the factors related to engaging in physical leisure activity, we compared background factors (gender, age, education, disease duration, HY stages, and LEDD) between the physical leisure activity engagement group (PLA-EG) and physical leisure activity non-engagement group (PLA-NEG) in patients with PD. There were no differences between the two groups in background factors. Table 1 shows the demographics of the PLA-EG and PLA-NEG.

\subsection{Non-Physical Leisure Activity}

In the HC group, 22 individuals (88\% of the total) were engaging in non-physical leisure activity. On the other hand, in the PD group, only 15 patients (47\%) were doing so. The PD group showed a significantly lower frequency of non-physical leisure activity than the HC group $\left(\chi^{2}(1, N=57)=10.42, p=0.002\right)$. Participants reported the following non-physical leisure activities: PD: reading, drawing pictures, writing a novel, gardening, etc.; HC: choir, tea ceremony, the game of "go", volunteering, reading, etc. In order to examine the factors related to the engagement of non-physical leisure activity, we compared background factors (gender, age, education, disease duration, HY stages, and LEDD) between the non-physical leisure activity engagement group (non-PLA-EG) and non-physical leisure activity non-engagement group (non-PLA-NEG) in patients with PD. There were no differences between the two groups in background factors. Table 2 shows the demographics of the non-PLA-EG and non-PLA-NEG.

Table 1. Demographic variables of physical leisure activity engagement and non-engagement groups.

\begin{tabular}{cccc}
\hline Demographic Variable & PLA-EG $(\mathrm{n}=14)$ & PLA-NEG $(\mathrm{n}=18)$ & $p$-value \\
\hline Gender (M/F) & $4 / 10$ & $6 / 12$ & 0.541 \\
Age (years) & $67.4(7.0)$ & $68.2(7.9)$ & 0.749 \\
Education (years) & $12.4(1.6)$ & $11.8(1.7)$ & 0.322 \\
Disease duration (years) & $5.8(4.8)$ & $5.5(4.7)$ & 0.866 \\
HY stage (II/III) & $3 / 11$ & $6 / 12$ & 0.457 \\
LEDD (mg) & $306.5(210.7)$ & $334.9(159.3)$ & 0.678 \\
\hline
\end{tabular}

All values represent mean (SD). $p$-values have been calculated using independent sample t-tests for parametric variables and $\chi^{2}$ tests for categorical variables. PLA-EG, physical leisure activity engagement group; PLA-NEG, physical leisure activity non-engagement group; LEDD, L-dopa equivalent daily dose; HY, Hoehn and Yahr.

\subsection{Relationship of Physical Leisure Activity with Cognitive Functions and Emotional Symptoms in Patients with PD}

Table 3 shows the results for group comparisons of cognitive functions and emotional symptoms. Patients in the 
Table 2. Demographic variables of non-physical leisure activity engagement and non-engagement groups.

\begin{tabular}{cccc}
\hline Demographic Variable & non-PLA-EG $(\mathrm{n}=15)$ & non-PLA-NEG $(\mathrm{n}=17)$ & $p$-value \\
\hline Gender (M/F) & $6 / 9$ & $4 / 13$ & 0.267 \\
Age (years) & $68.3(6.7)$ & $67.5(8.2)$ & 0.767 \\
Education (years) & $12.0(1.5)$ & $12.1(1.8)$ & 0.910 \\
Disease duration (years) & $6.8(4.4)$ & $4.6(4.7)$ & 0.183 \\
HY stage (II/III) & $4 / 11$ & $5 / 12$ & 0.863 \\
LEDD (mg) & $331.0(180.7)$ & $308.9(199.0)$ & 0.738 \\
\hline
\end{tabular}

All values represent mean (SD). $p$-values have been calculated using independent sample t-tests for parametric variables and $\chi^{2}$ tests for categorical variables. non-PLA-EG, non-physical leisure activity engagement group; non-PLA-NEG, non-physical leisure activity non-engagement group; LEDD, L-dopaequivalent daily dose; HY, Hoehn and Yahr.

Table 3. Cognitive and emotional variables of physical leisure activity engagement and non-engagement groups.

\begin{tabular}{|c|c|c|c|c|}
\hline Measure & & PLA-EG $(n=14)$ & PLA-NEG $(\mathrm{n}=18)$ & $p$-value \\
\hline \multicolumn{5}{|c|}{ Cognitive variables } \\
\hline \multirow[t]{4}{*}{ WCST } & Categories Completed & $5.4(1.6)$ & $4.2(2.6)$ & 0.134 \\
\hline & Total Errors & $54.8(20.7)$ & $30.2(23.3)$ & 0.519 \\
\hline & Perseverative Errors & $11.1(6.7)$ & $10.8(6.8)$ & 0.897 \\
\hline & Non-perseverative Errors & $13.7(14.5)$ & $19.4(17.2)$ & 0.347 \\
\hline \multirow[t]{3}{*}{ TMT } & Part A time (s) & $58.4(26.7)$ & $83.7(61.8)$ & 0.163 \\
\hline & Part B time (s) & 109.9 (41.8) & $189.7(122.4)$ & $0.017^{*}$ \\
\hline & Part B-Part A time (s) & $51.6(20.0)$ & $106.1(76.1)$ & $0.009^{* *}$ \\
\hline \multirow[t]{2}{*}{ DST } & Forward & $7.3(2.0)$ & $7.5(2.4)$ & 0.818 \\
\hline & Backward & $5.0(1.2)$ & $5.1(1.6)$ & 0.910 \\
\hline \multirow[t]{2}{*}{ VFT } & Letter [Ka] & $9.7(3.3)$ & $8.9(3.2)$ & 0.483 \\
\hline & Category [animal] & $17.3(6.6)$ & $15.4(6.5)$ & 0.435 \\
\hline \multirow[t]{2}{*}{ JVLT } & Immediate Recall Total & $31.6(8.2)$ & $29.0(7.1)$ & 0.350 \\
\hline & Delayed Recall & $12.1(3.4)$ & $12.1(2.3)$ & 0.969 \\
\hline \multicolumn{5}{|c|}{ Emotional variables } \\
\hline HAMD-17 ${ }^{\text {a) }}$ & & $2.1(2.4)$ & $2.7(2.0)$ & 0.463 \\
\hline Apathy Scale & & $9.6(5.4)$ & $10.9(6.1)$ & 0.510 \\
\hline
\end{tabular}

All values represent mean (SD). ${ }^{*} p<0.05 .{ }^{* *} p<0.01$. $p$-values have been calculated using independent sample t-tests for parametric variables and $\chi^{2}$ tests for categorical variables. a) Mann-Whitney U-test used because of non-normality of the variable. PLA-EG, physical leisure activity engagement group; PLA-NEG, physical leisure activity non-engagement group; WCST, Wisconsin Card Sorting Test; TMT, Trail Making Test; DST, Digit Span Test; VFT, Verbal Fluency Test; JVLT, Japanese Verbal Learning Test; HAMD-17, 17-item version of Hamilton Depression Rating Scale.

PLA-NEG performed worse than those in the PLA-EG on TMT part B and TMT part B minus part A (B-A), but the two groups did not differ significantly in the other neuropsychological tests and emotional symptoms.

\subsection{Relationship of Non-Physical Leisure Activity with Cognitive Functions and Emotional Symptoms in Patients with PD}

Table 4 shows the results of group comparisons of cognitive functions and emotional symptoms. Patients of the non-PLA-NEG scored higher than those of non-PLA-EG on the Apathy Scale, but the two groups did not differ significantly with regard to the other neuropsychological tests and emotional symptoms. 
Table 4. Cognitive and emotional variables of non-physical leisure activity engagement and non-engagement groups.

\begin{tabular}{|c|c|c|c|c|}
\hline Measure & & non-PLA-EG $(\mathrm{n}=15)$ & non-PLA-NEG $(\mathrm{n}=17)$ & $p$-value \\
\hline \multicolumn{5}{|c|}{ Cognitive variables } \\
\hline \multirow[t]{4}{*}{ WCST } & Categories Completed & $4.7(2.4)$ & $4.8(2.1)$ & 0.888 \\
\hline & Total Errors & $29.9(22.7)$ & 25.5 (21.9) & 0.602 \\
\hline & Perseverative Errors & $11.9(5.9)$ & $9.8(7.4)$ & 0.392 \\
\hline & $\begin{array}{l}\text { Non-perseverative } \\
\text { Errors }\end{array}$ & $17.9(17.2)$ & $15.7(15.3)$ & 0.717 \\
\hline \multirow[t]{3}{*}{ TMT } & Part A time (s) & $61.4(32.4)$ & 82.5 (61.7) & 0.246 \\
\hline & Part B time (s) & $129.0(61.7)$ & $177.6(126.4)$ & 0.172 \\
\hline & Part B-Part A time (s) & $67.6(34.2)$ & $95.1(80.8)$ & 0.214 \\
\hline \multirow[t]{2}{*}{ DST } & Forward & $7.9(2.3)$ & $6.9(2.0)$ & 0.179 \\
\hline & Backward & $5.3(1.5)$ & $4.8(1.3)$ & 0.376 \\
\hline \multirow[t]{2}{*}{ VFT } & Letter [Ka] & $9.6(3.1)$ & $8.9(3.4)$ & 0.574 \\
\hline & Category [animal] & $18.3(5.5)$ & $14.5(6.9)$ & 0.099 \\
\hline \multirow[t]{2}{*}{ JVLT } & Immediate Recall Total & $29.9(8.2)$ & $30.3(7.3)$ & 0.896 \\
\hline & Delayed Recall & $12.6(2.4)$ & $11.7(3.0)$ & 0.341 \\
\hline \multicolumn{5}{|c|}{ Emotional variables } \\
\hline HAMD-17 ${ }^{\mathrm{a}}$ & & $1.6(0.73)$ & $3.2(2.7)$ & 0.076 \\
\hline Apathy Scale & & $8.2(4.14)$ & $12.2(6.4)$ & $0.045^{*}$ \\
\hline
\end{tabular}

All values represent mean (SD). ${ }^{*} p<0.05 .{ }^{* *} p<0.01$. $p$-values have been calculated using independent sample t-tests for parametric variables and $\chi^{2}$ tests for categorical variables. a) Mann-Whitney U-test used because of non-normality of the variable. nonPLA-EG, non-physical leisure activity engagement group; non-PLA-NEG, non-physical leisure activity non-engagement group; WCST, Wisconsin Card Sorting Test; TMT, Trail Making Test; DST, Digit Span Test; VFT, Verbal Fluency Test; JVLT, Japanese Verbal Learning Test; HAMD-17, 17-item version of Hamilton Depression Rating Scale.

\section{Discussion}

The purpose of this study was to investigate how habitual leisure-time activities (both physical and non-physical leisure activities) may impact cognitive functions and emotional responses in patients with PD. We confirmed that compared to healthy controls, patients with PD performed both physical and non-physical leisure activities less frequently. Moreover, we found that patients with PD who participated in physical leisure activities performed better on the TMT. Patients who participated in non-physical leisure activities were less apathetic than patients who did no non-physical leisure activities.

While previous studies have reported that engaging in physical leisure activity is associated with age, gender, physical ability, and disease severity [5] [6], we found no differences in background factors between the two groups. The patients in the present study had a narrow range of HY stages and ages. Therefore, future studies will need to include patients with a wide range in disease severity and age to further validate our present findings.

Few studies have focused on the potential relationship between habitual leisure-time activities and non-motor symptoms in patients with PD. It is apparent that because of motor symptoms, patients with PD have a low frequency of habitual leisure-time activities compared to healthy controls. However, among healthy adults, retirement is associated with an increase in physical leisure activity [3]. Therefore, the fact that patients with PD showed a low frequency of habitual leisure-time activities in the present study leads to two possible interpretations. First, motor symptoms associated with PD may actively discourage habitual leisure-time activities. Second, the onset of PD may coincide with the time when retired populations typically begin leisure activities, and patients with PD might miss the opportunity to develop habits related to leisure-time activities.

The TMT measures psychomotor speed, attention, sequencing ability, mental flexibility, visual scanning, motor speed, and visual attention, as well as simple motor and spatial skills. Specifically, the TMT-B measures 
alternating attention, cognitive flexibility, and executive function because it requires the capacity to switch from one stimulus to another. In addition, the TMT (B-A) is able to accurately evaluate executive functions, such as the ability to switch attention, in patients with PD because it eliminates the influence of motor speed. Therefore, the results from this study suggest that the capacity of alternating attention (attention-switching function) declines under a dual-task situation among patients of the PLA-NEG compared to those of the PLA-EG. The capacity of executive and attention function declines with ageing, and such decreased ability is believed to result in an increased risk of falls among the elderly [7]. Furthermore, a study on patients with PD has suggested that a decline in executive function and attention-switching function leads to falls [8]. Previous studies have shown that the performance on the TMT (B-A) is related to physical function in elderly individuals [9] [10], such that participates who performed well on TMT (B-A) also had excellent performance on complex walking tasks requiring attention [9]. From the above results, we propose that engaging in a physical leisure activity may prevent the decline in the attention-switching function under a dual-task situation and may indirectly decrease the risk of falls in patients with PD.

Non-physical leisure activities may be associated with reduced apathy, although the reason for this is unclear. Apathy has been reported to be highly frequent among patients with PD [11] [12], and performing non-physical leisure activity may reduce apathy in these patients. In addition, increasing leisure-time activities during old age is very important for quality of life (QOL). Because leisure-time activities can encourage active lifestyles and social interactions, patients with PD will likely benefit from participating in these activities. Recent advances in treatment have led to dramatic improvements in motor symptoms in patients with PD. Nevertheless, the patients' QOL remains independent of motor symptoms. Unfortunately, some patients experience severe non-motor symptoms even when their motor symptoms are adequately controlled. Therefore, to improve the quality of support for patients with PD, medical practitioners must also focus on lifestyle, living environment, and social well-being, in addition to addressing motor symptoms. There are some limitations in the present study. First, we did not examine the type, level, and period of activity. Second, we did not evaluate the patients' subjective QOL.

\section{Conclusion}

The present study suggests that habitual leisure-time activities may modulate cognitive functions and apathetic attitudes in patients with PD. From a therapeutic view, reducing sedentary lifestyles and promoting habitual leisure-time activities might be beneficial in preventing the cognitive decline and apathy.

\section{Acknowledgements}

The authors wish to thank the participants and their families. This work was supported by JSPS KAKENHI, Grant-in-Aid for JSPS Fellows, 242666.

\section{References}

[1] Speelman, A.D., van de Warrenburg, B.P., van Nimwegen, M., Petzinger, G.M., Munneke, M. and Bloem, B.R. (2011) How Might Physical Activity Benefit Patients with Parkinson Disease? Nature Reviews Neurology, 7, 528-534. http://dx.doi.org/10.1038/nrneurol.2011.107

[2] Wang, H.X., Karp, A., Winblad, B. and Fratiglioni, L. (2002) Late-Life Engagement in Social and Leisure Activities Is Associated with a Decreased Risk of Dementia: A Longitudinal Study from the Kungsholmen Project. American Journal of Epidemiology, 155, 1081-1087. http://dx.doi.org/10.1093/aje/155.12.1081

[3] Barnett, I., van Sluijs, E.M. and Ogilvie, D. (2012) Physical Activity and Transitioning to Retirement: A Systematic Review. American Journal of Preventive Medicine, 43, 329-336. http://dx.doi.org/10.1016/j.amepre.2012.05.026

[4] Iwasa, H., Yoshida, Y., Kai, I., Suzuki, T., Kim, H. and Yoshida, H. (2012) Leisure Activities and Cognitive Function in Elderly Community-Dwelling Individuals in Japan: A 5-Year Prospective Cohort Study. Journal of Psychosomatic Research, 72, 159-164. http://dx.doi.org/10.1016/j.jpsychores.2011.10.002

[5] Dontje, M.L., de Greef, M.H., Speelman, A.D., et al. (2013) Quantifying Daily Physical Activity and Determinants in Sedentary Patients with Parkinson's Disease. Parkinsonism \& Related Disorders, 19, 878-882. http://dx.doi.org/10.1016/j.parkreldis.2013.05.014

[6] van Nimwegen, M., Speelman, A.D., Hofman-Van Rossum, E.J., et al. (2011) Physical Inactivity in Parkinson’s Disease. Journal of Neurology, 258, 2214-2221. http://dx.doi.org/10.1007/s00415-011-6097-7

[7] Mirelman, A., Herman, T., Brozgol, M., et al. (2012) Executive Function and Falls in Older Adults: New Findings 
from a Five-Year Prospective Study Link Fall Risk to Cognition. PLoS One, 7, Article ID: e40297. http://dx.doi.org/10.1371/journal.pone.0040297

[8] Hirota, C., Watanabe, M., Sun, W., et al. (2010) Association between the Trail Making Test and Physical Performance in Elderly Japanese. Geriatrics \& Gerontology International, 10, 40-47. http://dx.doi.org/10.1111/j.1447-0594.2009.00557.x

[9] Vazzana, R., Bandinelli, S., Lauretani, F., et al. (2010) Trail Making Test Predicts Physical Impairment and Mortality in Older Persons. Journal of the American Geriatrics Society, 58, 719-723. http://dx.doi.org/10.1111/j.1532-5415.2010.02780.x

[10] Yogev, G., Giladi, N., Peretz, C., Springer, S., Simon, E.S. and Hausdorff, J.M. (2005) Dual Tasking, Gait Rhythmicity, and Parkinson's Disease: Which Aspects of Gait Are Attention Demanding? European Journal of Neuroscience, 22, 1248-1256. http://dx.doi.org/10.1111/j.1460-9568.2005.04298.x

[11] Starkstein, S.E., Mayberg, H.S., Preziosi, T., Andrezejewski, P., Leiguarda, R. and Robinson, R.G. (1992) Reliability, Validity, and Clinical Correlates of Apathy in Parkinson's Disease. The Journal of Neuropsychiatry and Clinical Neurosciences, 4, 134-139.

[12] Starkstein, S.E., Merello, M., Jorge, R., Brockman, S., Bruce, D. and Power, B. (2009) The Syndromal Validity and Nosological Position of Apathy in Parkinson's Disease. Movement Disorders, 24, 1211-1216.

http://dx.doi.org/10.1002/mds.22577 
Scientific Research Publishing (SCIRP) is one of the largest Open Access journal publishers. It is currently publishing more than 200 open access, online, peer-reviewed journals covering a wide range of academic disciplines. SCIRP serves the worldwide academic communities and contributes to the progress and application of science with its publication.

Other selected journals from SCIRP are listed as below. Submit your manuscript to us via either submit@scirp.org or Online Submission Portal.
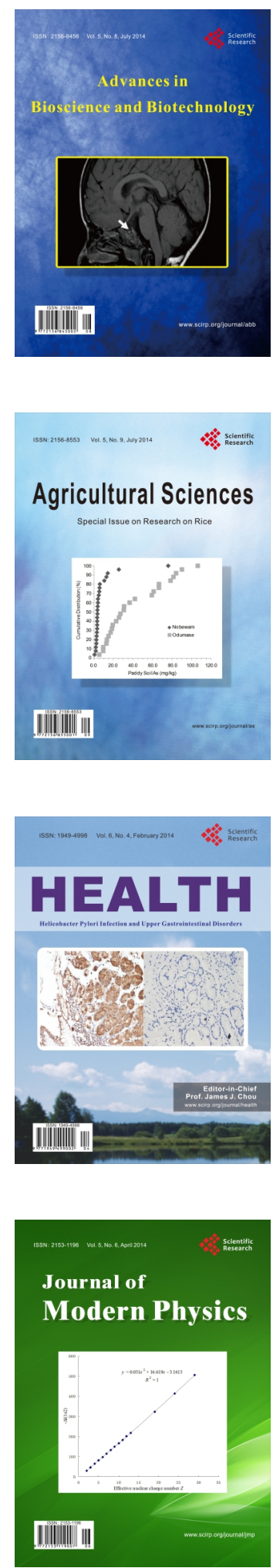
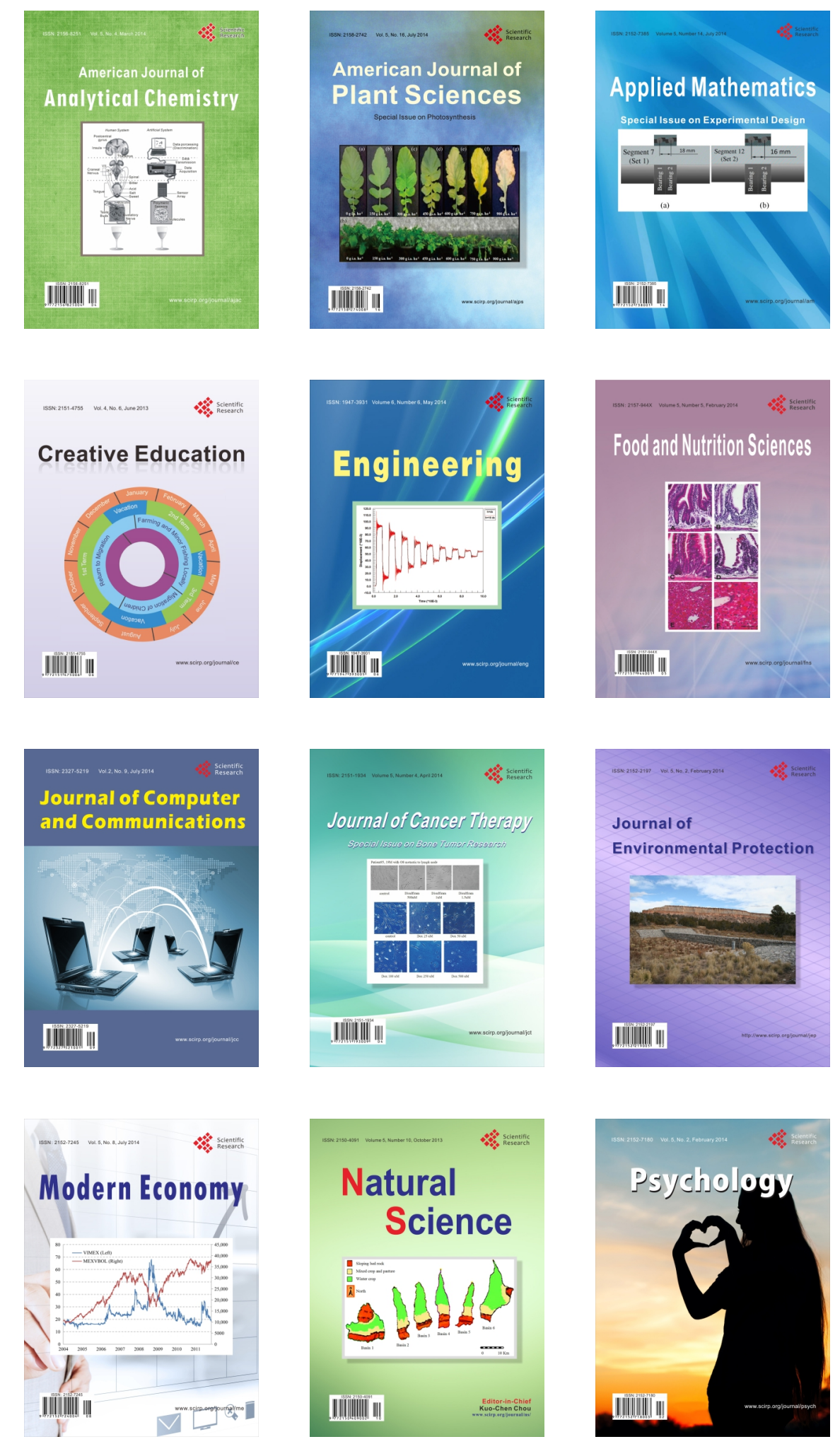